\title{
Complex relationships between adolescents and their sleep (sleep patterns, use of new media, and impact on next day's activity)
}

\author{
Interview with S. Royant-Parola ${ }^{1,2,3}$ by S. Legris ${ }^{4,5}$ \\ 1 Morphée Network, 2 Grande Rue, 92380 Garches, France \\ 2 Nightingale Hospital, Garches Castle, 92380 Garches, France \\ ${ }^{3}$ Antoine-Béclère Hospital, AP-HP, Center for Sleep Medicine, Clamart, France \\ 4 Specialist in Dento-facial Orthopedics, Private practice \\ ${ }^{5}$ Lecturer DIUTOSSAOS Paris, France
}

\begin{abstract}
During adolescence, sleep changes physiologically and biologically. It is during this period of life that development and learning takes place. However, new teenager sleep behaviors appear. A decrease in weekly sleep time and a jet lag during weekends contribute to the deterioration of teenager's sleep. The use of new social media plays a detrimental role, with a strong negative correlation between time spent on those and sleep time. Teenagers suffering from sleep deprivation develop well-identified consequences such as diurnal fatigue, lack of attention, more anxiety, poor self-esteem, and also an increased risk of obesity and depression. Teenage sleep is a real public health issue.
\end{abstract}

\section{KEYWORDS}

Teenagers, sleep, sleep deprivation, screens, social media, sleep new behaviors

\section{First, how old is the teenager?}

Theoretically, a teenager is anyone aged 13-18 years and is biologically defined by sexual maturation, the date of the first period for girls, and a number of characteristics in boys, according to the Tanner scale, which precisely defines bone maturation and specific age.
In practice, adolescent behaviors continue beyond the particular age group of 13-18 years. Does adolescence not end when the young person begins to take charge of his or her own life, which can be anywhere from 20 to 22 or even 25 years of age? The most complex period is that between 15 and 18 years because it is in this

Address for correspondence:

Sylvie Royant-Parola - Morphée Network - 2 Grande Rue - 92380 Garches

E-mail: dr.royant-parola@reseau-morphee.fr

Article received: 07-03-2017. Accepted for publication: 08-01-2017.

This is an Open Access article distributed under the terms of the Creative Commons Attribution License (http://creativecommons.org/licenses/by/4.0), which permits unrestricted use, distribution, and reproduction in any medium, provided the original work is properly cited. 
period that maturation occurs simultaneously with other challenges, such as those in social life, sexual development, and discovering sexual identity.

As a result, sleep tends to suffer because young persons have an objectively busy schedule. Classes often exceed 35 h a week, not including extracurricular activities and social life. Teenagers' social lives are important to them (friends and the need to be with each other and to belong to a group). Thus, parents have a hard time setting limits.

\section{Can you define the criteria for normal sleep in adolescents?}

The normal sleep of teenager is a sleep that has matured compared with that of young children. That is, it is structured like that of adults in 90-min cycles. One of the components, deep sleep, which is extremely long in prepubescents, decreases to be within the adult range of approximately 100 minutes per night. Physiological and biological characteristics of adolescents foster the tendency to go to bed later and get up later, whereas the amount of sleep they need increases, especially after the age of 15 years. According to international recommendations, sleep time for students aged $<17$ years is $8-10$ hours per night. Of course, in most cases with the exception of holidays, teenagers never achieve this duration, so they are always a little sleep-deprived. As a result, teenagers tend to be quite drowsy in the afternoon. Moreover, they still need a lot of sleep due to major maturation phases, in terms of their psychological and cognitive development as well as their education, especially in middle or high school. This describes the normal sleep of the teenager.

\section{During school time, adolescents fall asleep later and later but get up earlier and earlier, creating sleep deprivation. What factors contribute to this? What are the consequences?}

One factor to consider is the pace of parents who generally come home later, especially in urban areas compared with more rural areas. In large cities, parents often come home around $8 \mathrm{pm}$. Therefore, dinner is prepared late around 8:30-9:00 pm, which has been highlighted as a major problem (SLEEP 2017 Convention, Boston). In fact, the later the dinner, the closer the adolescent is to bedtime, when there is an increase in melatonin level, increasing the risk of insulin resistance created by the association "meals-high melatonin" with additional consequences on weight.

Late bedtime is a current trend in society and adolescents tend to exacerbate this phenomenon, especially considering that they are very much in demand mainly for social activities, spending time with friends and partners and often spending a lot of time on social media. We conducted a survey in 2013-2014 with 776 middle school students from 6th to 3rd grade (equivalent to the 6th to 9th grade in the USA) in Paris (mean age $12.4 \pm 1.2$ years) and assessed the use of social media and its effect on the sleep and daytime functioning of young people. This study shows that home devices are important for $98.3 \%$ of teenagers having an internet connection. A total of $42.7 \%$ young people have a computer in their room, $26.4 \%$ have a personal television in their room, and $85.2 \%$ have a personal mobile phone; $66.7 \%$ of middle school students have a smartphone, which has an internet 
connection. In addition, teenagers are often multi-tasking: listening to music, watching videos, performing internet searches, and using social networks. Moreover, these communication tools, which are an integral part of their social lives, have an effect on their rhythms by giving off light at a time when light should be dimmed. A specific light characteristic of these new displays, particularly Retina displays, is a blue light spectrum prominence (around $460 \mathrm{~nm}$ ). This intense light held very close to the eyes affects the retina by blocking melatonin secretion, thereby shifting their sleep pattern with a waking effect and disorganizing the circadian rhythm. Television screens do not produce the same effect because they are usually much further away from the eyes.

In conclusion, adolescents experience a dual shift in sleep patterns, physiological on the one hand and social on the other, reinforcing each other at this time in their lives.

\section{Can you clarify the relationship between sleep and weight?}

At the Boston Sleep Convention, the complicated relationship between sleep and weight was discussed. In fact, sleep position during the nycthemeral period, its regularity, and its duration have an effect on weight. Sleeping late promotes metabolic disturbances. In fact, reduced sleep duration related to late bedtime in teenagers leads to sleep deprivation and causes difficulties getting up in the morning. This sleep deprivation will modify insulin secretion as well as leptin and ghrelin secretion, contributing to the creation of a state of insulin resistance and promoting higher food intake and less food breakdown during digestion. In effect, teenagers are extremely susceptible to weight gain.

Research on the mechanisms have shown that irregular rhythm with a later phase shift, notably meal and sleep schedules, will have similar consequences on the same three parameters: insulin, leptin, and ghrelin secretion. However, teenagers with chronic sleep deprivation during the week sleep more on weekends, but with a later bedtime and wake-up time. It should be noted that this behavior is not limited to one night but generally affects two nights (Friday and Saturday nights). Therefore, a real social jetlag is established, and this "week/weekend" irregularity introduces another parameter that reinforces metabolic disorders.

\section{Many teenagers spend time in front of a screen every day of the week? What is the impact?}

Time spent in front of screens is important both before going to bed and when in bed; worse, some teenagers spend time on social networks at night via appointments they set up to chat at 2:00-3:00 am.

In our study, $52.6 \%$ of middle school students spend over an hour after dinner on a console, tablet, or computer, including $14.7 \%$ who spend $>2 \mathrm{~h}$ in front of a screen. Once in bed, 51.7\% regularly use an electronic device (33.1\% of which is a telephone) with on-screen activities for $26.6 \%$ of them: SMS, social networks, videos, and television. Throughout the night, 15.3\% of teenagers send SMSs, $11 \%$ connect on social networks, $6.1 \%$ play on the internet, and $22.1 \%$ of them spend more than an hour doing this. In most cases $(73.9 \%)$, these activities happen 
spontaneously, whereas $21.6 \%$ organize them at bedtime, with $15.5 \%$ waiting until everyone else is asleep to play or chat and $10.6 \%$ setting an alarm to wake up to go online. Often, parents are unaware about these activities.

This delays the time it takes to fall asleep and reduces overall sleep time.

In conclusion, a strong negative correlation between screen time and sleep time was found. As for the deterioration in sleep quality associated with the use of new media, it is associated with higher anxiety level, depression, and low self-esteem.

\section{Could you explain the psychological consequences of this sleep deprivation?}

Adequate sleep is a source of psychological well-being. Sleep deprivation weakens the individual as a whole on different levels. Effects are seen mainly on mood and concentration, ability to think or imagine, difficulty with decision-making, impaired judgment, difficulty with memorization, decreased ability to perform somewhat complex tasks, physical or intellectual. This deterioration is proportional to sleep deprivation. Studies have shown that sleep deprivation for 20 hours can lead to a drop in performance similar to having $1 \mathrm{~g}$ of alcohol in the blood. Repeated chronic deprivation leads to a weakening of the individual's adaptation mechanisms with a risk of depressive decompensation.

Those who sleep little tend to be in a worse mood both in the morning and throughout the day. Irritability is also associated with sad or sullen mood. As a result, they are much more reactive children or teenagers with much less control over their emotions and reactions, which will not be proportionate to the events experienced. This can be a problem to their social life at school.

\section{Can you define delayed sleep phase?}

Delayed sleep phase has a very precise definition; it is not just late bedtime. We talk about delayed sleep phase when systematically at night, sleep is not possible before $1 \mathrm{am}$ and is associated with difficulty in waking up, with the following difficulty levels: difficulty in waking up, waking up without help is almost impossible, and waking up is almost impossible even with help, resulting in missed lessons and serious school issues, if parents do not intervene. In the delayed sleep phase, everything is out of phase, i.e., early sleep is not possible because of an out-of-sync biological clock. Of course, it is necessary to enter a therapeutic process entailing the readjustment of rhythms by possible chronotherapy with strong involvement of the teenagers and parents, which is essential to obtain a positive result. Parents are often tolerant with their teenagers who cannot get up to go to school. It is not uncommon to see parents waiting for 6 months and young people coming in for a consultation only in April or May. It is difficult to take care of them at this time as soon it will be school vacation and the teenagers will sleep very well during the summer and will not be motivated to recalibrate their sleep patterns. To be effective, the consultation should take place in November or December.

\section{Phase shift? Delayed sleep phase? Are there warning signs?}

I think there is a warning signal as soon as the young person really has 
difficulty in waking up. Of course, this can happen occasionally, but when a young person fails to get up 1-2 times a week systematically, this is a serious sign for parents and requires their attention as it signals incorrect functioning and a loss of the teenager's sleep routine.

A 2010 study by the Sleep journal showed that when parents give instructions to their teenager, even in the older age group of 16-19 years, young people take this into account; $80 \%$ of them show discontent, but they go to bed at around the hour given by the parents and $10 \%$ go to bed an hour later, but still respect the framework. Only 10\% absolutely did not comply with the request. This highlights that giving instructions to a teenager will help them. The study questioning both teenagers and parents showed that even if parents' instructions are a bit of a nuisance to young people, they recognize that this demonstrates parents' interest in them. Therefore, they accept the concept.

\section{We did not discuss the other behaviors: alcohol, tobacco, drugs. Is it common? Is the use of these substances increasing? Decreasing? What is the impact on the phenomena we have described?}

Alcohol use remains fairly stable. However, alcohol consumption is starting at younger ages than before and excessive and abnormal use is observed in teenagers who consume it.

On the other hand, cannabis use is becoming commonplace. It is used by a large number of young people almost systematically at bedtime. Indeed, cannabis has a soothing function and provides some relief to anxious young people going through difficult times! But on sleep, it acts on one of the stages of melatonin synthesis, delaying its secretion and increasing the delayed sleep phase. Very often, among young people who exhibit delayed sleep phase, there is cannabis use. In therapy, it is necessary to treat cannabis use separately, which complicates the management.

\section{Delayed sleep phase syndrome and adolescent insomnia.}

In delayed sleep phase syndrome, young people fall asleep late and wake up late. Why do young people reach such extremes?

It is important to distinguish between an teenager who occasionally has a late bedtime and a late wakeup time with difficulties in waking up and one who is in a process wherein sleep is systematically delayed and waking up gets increasingly difficult or even impossible. In true delayed sleep phase syndrome, two-third of teenagers suffer from depression, which requires assessing both the adolescent's psychological condition and their relationships. Indeed, in adolescents, depression is a serious problem with risks, considering that it is the leading cause of suicide among 15-25-year-olds. It is often expressed in a hidden form: they are not depressed in the traditional sense. More frequently, adolescents isolate themselves, withdraws into themselves, fail to accomplish tasks assigned to them, and do not talk to their parents or friends. However, this is an age with wild extremes, and for the young person who does not find a solution, suicide seems a possible 
alternative. It is important for practitioners to consider this hypothesis when treating adolescents.

Sleep apnea syndrome, which exists in adolescents, although it is also accompanied by real fatigue in the morning and attention difficulties during the day, is much rarer than depression with or without delayed sleep phase. Therefore, it is so important to perform a psychiatric and psychological assessment of a teenager with sleep disorders.

In conclusion, in delayed sleep phase, there is usually at least some anxiety, but very often depression.

\section{In this process, what is the impact of new behaviors on internet-connected adolescents?}

Teenagers who are constantly on their smartphones tend to lose touch with real life and contact with others, preferring to chat online instead of meeting their friends and family and having a face-to-face conversation, which can have a positive effect on the teenager. Some prefer or even only use social media to keep in touch, without being in a relational exchange; this highlights their discomfort in relating with others. However, most often, the smartphone is a tool that can exacerbate this link with some friends. In this way, teenagers continue the relationship in a text message discussion and have difficulty ending the conversation.

The telephone, and more particularly the smartphone, functions as a tool for socialization, exchanges, sharing, and contact with others, friends, confidants, and strangers on the internet and becomes "an extension of oneself" in communication.

\section{Is there a link between these new behaviors of internet-connected adolescents and social anxiety?}

It is not these new behaviors that provoke agoraphobia or even social anxiety; however, among people with social phobias, the use of social media is particularly interesting: while keeping them safe from contact and without disturbing their lives, a relationship exists.

For example, it has been found that people with autism are more comfortable with this type of media because they manage to maintain a relationship in this manner when they would normally be very uncomfortable directly dealing with people and would remain isolated.

\section{Is the teenager's schooling disrupted by these new behaviors?}

Teenagers' schooling is disrupted as soon as they go to bed very late to get up in the morning, which creates sleep deprivation. Their attention, concentration, and learning, all that is related to their schooling, will be impaired. Because they are less alert, it will be difficult for them to follow lessons properly

with lapses in attention and sometimes, there may be pseudo-hyperactive behaviors. It is a reactive hyperactivity characterized by attention disorders, an attitude that is becoming more and more frequent. Teenagers with sleep deprivation may be more irritable and more absent-minded.

Some teenagers sleep only 5 hours a night over a long period of time. The risk is that they gradually stop going to school from 1 or 2 times randomly to 2-3 days a week and then not at all. 


\section{In conclusion, the adolescent's sleep is therefore a real public health issue. What are your proposals?}

Is it desirable to arrange schedules? Specialists wonder about starting classes later. In English-speaking countries, courses start at 7:30 am, which is rare in our country. Our high school students start at 8 am, more often at 9 am. The solution is not necessarily in changing schedules but rather in the sequence. A teenager who is tired and takes a nap when he or she gets home at around 5 or $6 \mathrm{pm}$ will not go to bed early in the evening. On the contrary, the late nap, resulting in a decrease in sleep pressure, reinforces an increasingly later bedtime. So, these are the principles of sleep that should be taught to children and adolescents.

As far as possible, it is necessary to explain sleep physiology before adolescence: what is sleep, what is it for, and how should one sleep? Therefore, by the time they become teenagers, they would have acquired knowledge about sleep, both about sleep needs and inappropriate behaviors. Admittedly, teenagers are always in transgression at some point, seeking to go beyond the limits, but they will understand the consequences of these decisions. They should be made aware of the importance of sleep, the fundamental role of sleep in being able to perform well in school, in sports, and with their friends and that in subjecting themselves to sleep deprivation, they are not going to save time. One has the impression that sleeping is a waste of time!

Also, even before the issue of school schedules, the establishment of a real educational program on sleep, like the one created for food, is essential. As sleep specialists, we have a role to provide the National Education Department with support materials, tools, and exercises to encourage exchanges, with the involvement of parents.

\section{REFERENCE DOCUMENTS}

1. Royant-Parola S., et al. Nouveaux médias sociaux, nou-veaux comportements de sommeil chez les adolescents. Encéphale (2017), http://dx.doi.org/10.1016/j. encep.2017.03.009.

2. Royant-Parola S. Le sommeil des adolescents, une priorité de santé publique. Médecine du Sommeil 2017;14:61-62.

3. www.reseau-morphee.fr 